\title{
Letter to Editor: Is Mitochondria Biogenesis and Neuronal Loss Prevention in Rat Hippocampus Promoted by Apigenin?
}

\author{
Salvatore Chirumbolo (iD
}

1. Department of Neuroscience, Biomedicine and Movement Sciences, Unit of Human Anatomy, University of Verona, Verona, Italy.

chtation: Chirumbolo, S. Is Mitochondria Biogenesis and Neuronal Loss Prevention in Rat Hippocampus Promoted by Apigenin? Basic and Clinical Neuroscience, 10(6), 541-544. https://doi.org/10.32598/bcn.10.6.541

https://doi.org/10.32598/bcn.10.6.541

\section{Highlights}

- Apigenin improves spatial working memory in Wistar rats.

- Apigenin promotes mitochondrial biogenesis by activating peroxisome Proliferator-activated receptor Gamma Coactivator 1-alpha (PGC-1 $\alpha$ ).

- Apigenin prevents neurodegeneration in rat hippocampus.

- The neurodegenerative damages are mediated by mitochondrial biogenesis.

- Apigenin prevents neurodegeneration by inducing PGC-1 $\alpha$.

\section{Plain Language Summary}

In this correspondence, a comment to a recent paper by Nikbakht et al., published in the latest issue of this journal is reported. The flavone apigenin can exert both its antioxidant potential via the usual enzymatic reactive oxygen species scavenging system and the mitochondrial biogenesis via the peroxisome Proliferator-activated receptor Gamma Coactivator 1-alpha (PGC-1 $\alpha$ ) mitochondrial Transcription Factor A (TFAM) Nuclear Respiratory factor 1 (NRF-1) pathway. The fascinating paper by Nikbakht et al., earns more insightful clues about the activity of apigenin in the prevention of the rat hippocampus neuronal loss caused by the A $\beta 25-35$ injection. 


\section{Introduction}

$\mathbf{T}$

he recent paper by Nikbakht et al., reported that the flavone apigenin (4',5,7-trihydroxyflavone) improves spatial working memory in adult male Wistar rats (Nikbakht et al., 2019). According to the authors, apigenin induces a protective effect against neurotoxicity caused by the amyloid fragment $\beta-25-35$ in a rodent Alzheimer disease model (Nikbakht et al., 2019). According to the authors, besides the antioxidant and anti-apoptotic properties of apigenin that may prevent neurodegenerative damages in the hippocampal area, further mechanisms can be addressed. In this circumstance, it can be suggested that changes in the levels of mitochondrial Transcription Factor A (TFAM), peroxisome Proliferator-activated Receptor Gamma Coactivator 1-alpha (PGC-1 $\alpha$ ), phospho-adenosine monophosphate (AMP)-activated protein kinase (pAMPK), AMPK, phospho-cAMP-responsive element binding protein (CREB), and nuclear respiratory factor 1 (NRF-1) proteins occur, particularly for the expression of PGC- $1 \alpha$ and the phosphorylation of CREB, which is a master tuner of the memory processes (Ashabi et al., 2012).

PGC-1 $\alpha$ and its downstream molecules NRF-1 and TFAM increase following a diet enriched in plant derived-flavones. In this circumstance, the mitochondrial biogenesis results in the prevention of hippocampal neuronal loss as a leading mechanism. Flavonoids are mild activators of reactive oxygen species, which should act as signaling molecules and moderate activators of proapoptotic signals with the complex role to tune the cellular survival machinery (Chirumbolo \& Bjørklund, 2017). Some pro-apoptotic signals are potent inducers of mitochondrial biogenesis. At the dose used by the authors, 50 $\mathrm{mg} / \mathrm{kg}$ by oral gavage every day, the flavone apigenin can induce the early expression of procaspase- 3 , which is crucial for the activation of mitochondrial biogenesis initiators, such as TFAM and NRF-1 (Kim, Ha, Yang, \& Son, 2018). In this perspective, a significant role is ruled by the PGC- $1 \alpha$, the expression of which is particularly stringent for mitochondrial biogenesis (Chen, Tao, Li, \& Yao, 2018; Niu, Tang, Ren, \& Feng, 2018).

Apigenin exerts its antioxidant role also by inducing the activation of PGC- $1 \alpha$, which in turn can attenuate hydrogen peroxide-induced apoptotic cell death by upregulating Nrf-2 via GSK3 3 inactivation mediated by activated p38 (Choi et al., 2017). This phenomenon would mean that apigenin can exert both its antioxidant potential via the usual enzymatic reactive oxygen species scavenging system and the mitochondrial biogenesis via the PGC$1 \alpha /$ TFAM/NRF-1 pathway. The fascinating paper by
Nikbakht et al., 2019. earns more insightful clues about the activity of apigenin in the prevention of the rat hippocampus neuronal loss caused by the A $\beta 25-35$ injection.

\section{Ethical Considerations}

\section{Compliance with ethical guidelines}

This manuscript contains original and new ideas, which are not submitted for publishing to other publications or published elsewhere. The corresponding and unique author made substantial contributions to the conception or design of the work. No ethical declarations about patients, psychology, and animals are applicable. References are properly cited within the text and in the reference list.

\section{Funding}

This research did not receive any specific grant from funding agencies in the public, commercial, or not-forprofit sectors.

\section{Acknowledgments}

A special thanks go to Anna-Maria Renda for her valuable technical advice.

\section{References}

Ashabi, G., Ramin, M., Azizi, P., Taslimi, Z., Alamdary, S. Z., Haghparast, A., et al., (2012). ERK and p38 inhibitors attenuate memory deficits and increase CREB phosphorylation and PGC-1a levels in A $\beta$-injected rats. Behavioural Brain Research, 232(1), 165-73. [DOI:10.1016/j.bbr.2012.04.006]

Chen, Z., Tao, S., Li, X., \& Yao, Q. (2018). Resistin destroys mitochondrial biogenesis by inhibiting the PGC-1a/NRF1/TFAM signaling pathway. Biochemical and Biophysical Research Commu nications, 504(1), 13-8. [DOI:10.1016/j.bbrc.2018.08.027] [PMID]

Chirumbolo, S., \& Bjørklund, G. (2017). PERM hypothesis: the fundamental machinery able to elucidate the role of xenobiotics and hormesis in cell survival and homeostasis. International Journal of Molecular Sciences, 18(1), 165. [DOI:10.3390/ijms18010165] [PMID] [PMCID]

Choi, H. I., Kim, H. J., Park, J. S., Kim, I. J., Bae, E. H., Ma, S. K., et al., (2017). PGC-1a attenuates hydrogen peroxide-induced apoptotic cell death by upregulating Nrf-2 via GSK3 $\beta$ inactivation mediated by activated p38 in HK-2 Cells. Scientific Reports, 7(1), 1-13. [DOI:10.1038/s41598-017-04593-w]

Kim, J. S., Ha, J. Y., Yang, S. J., \& Son, J. H. (2018). A novel non $\square$ apoptotic role of procaspase $\square 3$ in the regulation of mitochondria biogenesis Activators. Journal of Cellular Biochemistry, 119(1), 347-57. [DOI:10.1002/jcb.26186] 
Nikbakht, F., Khadem, Y., Haghani, S., Hoseininia, H., Hashemi, P., \& Jamali, N. (In Press). Protective role of apigenin against A $325-35$ toxicity via inhibition of mitochondrial cytochrome c release. Basic and Clinical Neuroscience. [DOI:10.32598/bcn.9.10.385]

Niu, Z., Tang, J., Ren, Y., \& Feng, W. (2018). Ropivacaine impairs mitochondrial biogenesis by reducing PGC-1a. Biochemical and Biophysical Research Communications, 504(2), 513-8. [DOI:10.1016/j. bbrc.2018.08.186] 
This Page Intentionally Left Blank 